\title{
Field-Trial Demonstration of Span Launch Power Optimization using a Subcarrier Non-
} Linearity Monitor

Petersen, Martin Nordal; Nielsen, Mads Lønstrup; Buron, Jakob Due

Published in:

Proc. of OFC 2005

Publication date:

2005

Document Version

Publisher's PDF, also known as Version of record

Link back to DTU Orbit

Citation (APA):

Petersen, M. N., Nielsen, M. L., \& Buron, J. D. (2005). Field-Trial Demonstration of Span Launch Power Optimization using a Subcarrier Non-Linearity Monitor. In Proc. of OFC 2005 (Vol. Paper OTuH2, pp. 170-172). IEEE.

\section{General rights}

Copyright and moral rights for the publications made accessible in the public portal are retained by the authors and/or other copyright owners and it is a condition of accessing publications that users recognise and abide by the legal requirements associated with these rights.

- Users may download and print one copy of any publication from the public portal for the purpose of private study or research.

- You may not further distribute the material or use it for any profit-making activity or commercial gain

- You may freely distribute the URL identifying the publication in the public portal 


\title{
Field-Trial Demonstration of Span Launch Power Optimization using a Subcarrier Non-Linearity Monitor
}

\author{
Martin Nordal Petersen, Mads Lenstrup Nielsen and Jakob Buron \\ Research Center COM, Technical University of Denmark, DK2800 Kgs. Lyngby. Denmark \\ mmp@com.dtu.dk
}

\begin{abstract}
This paper presents a 500-km field-trial demonstration of power optimization in a four span transmission link. A new non-linearity monitor method based on an in-band subcarrier was used to supervise the signal quality. 2004 Optical Society of America OCIS codes: (060.4510) Optical communications; (190.5940) Self-action effects; (190.5890) Scattering, stimulated
\end{abstract}

\section{Introduction}

Optical performance monitoring is becoming even more important as communication systems and networks are moving closer to full end-to-end transparency. The traditional intermediate $\mathrm{O} / \mathrm{E} / \mathrm{O} 3 \mathrm{R}$ regenerators (re-amplification, re-shaping and re-timing) that are found in many transmission systems make sure that signal degradation is limited at each node. However, in optical transparent networks any possible signal degradation will be carried on much further into the network and therefore making signal quality monitoring in transparent networks a key issue. Several methods have already been developed to monitor different signal parameters such as chromatic dispersion $[1,2]$, Optical Signal to Noise ratio (OSNR) [3] and polarization mode dispersion (PMD) [4].

These parameters are important to monitor in order to ensure high signal quality. Another relevant parameter is the launch power to the individual spans. Typically it is desirable to keep the launch power as high as possible to maximize the OSNR throughout transmission but still below the limit where fiber non-linearities will degrade the signal quality.

Fiber non-linearities limit the fiber launch power, but the specific limit depends on several different factors such as the type of fiber used, the bit rate, amplifier spacing, and the applied dispersion map. Even in today's point-to-point links it can be a difficult task to identify the optimal input power that provides minimum bit-error-rate (BER), and furthermore requires expensive BER test equipment as well as complicated measurements.

This paper reports on a $500-\mathrm{km}$ field-trial, which demonstrates a new method to optimize the launch power by adding a subcarrier tone to the baseband data. By establishing a correlation between the BER performance and the subcarrier RF power, we are able to minimize the BER, without prior knowledge of the data, by tuning the launch power to the link. The power of the subcarrier tone will in a simple way indicate the optimum launch power, corresponding to the best signal quality.

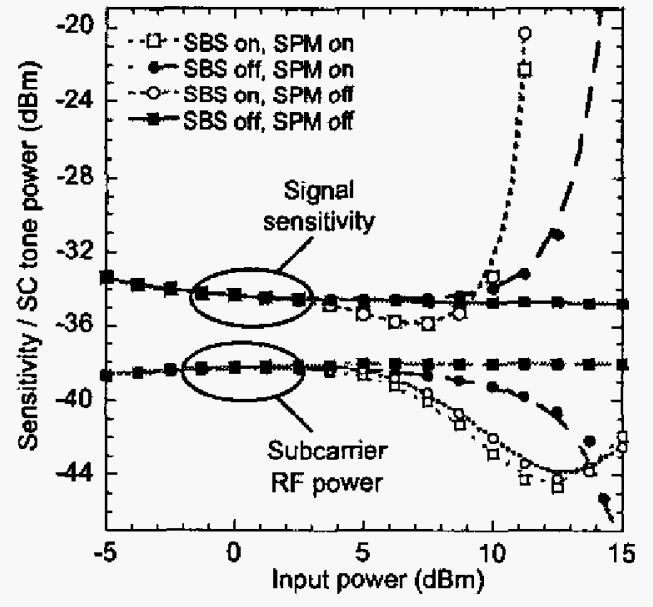

Fig. 1. Simulation results done in one 80 -km post compensated span for a) No SPM, with SBS, b) No SBS, with SPM c) no SBS, no SPM, and d) with both SBS and SPM effects included in the model. The figure shows the individual non-linear effects on the subcarrier RF power and the $10 \mathrm{~Gb} / \mathrm{s}$ data signal.

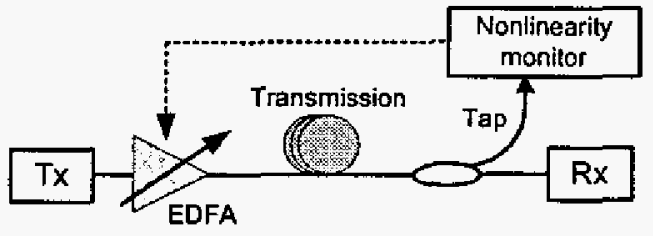

Fig 2. Figure showing the principle of operation of the nonlinearity monitor. The subcarrier tone is added at the transmitter and subsequently extracted to provide information about the non-linearities excited in the system. 


\section{OTuH2}

\section{Operational principles of the Non-linear monitor}

It is well-known that by adding a subcarrier to the baseband of the signal it is possible to monitor the accumulated chromatic dispersion of a signal $[1,2]$. However, we find that if the dispersion is assumed constant, the subcarier can also be used to monitor fiber non-linearities, as the detected tone RF power will fade according to the level of excited non-linearities in the system. This effect has been investigated though simulations (using VPI Transmission Maker), which show that the detected subcarrier RF power will be influenced by Stimulated Brillouin Scattering (SBS) as well as by Self Phase Modulation (SPM). The results from these simulations can be seen in Fig. 1. The 80$\mathrm{km}$ post-compensated span was studied in four different cases as the model allowed for independently switching on and off the effects of SBS and SPM. This provides a method for identifying which effect is the dominating one in terms of signal degradation as well as subcarrier RF fading.

Considering the results from the simulations in Fig. 1 it is clear that SBS is primarily responsible for the fading of the subcarrier, and that the impact of SPM is only significant in the absence of SBS. This is not surprising as SBS is a narrowband scattering effect that will influence the tone due to the narrow tone bandwidth, which is in the order of a few $\mathrm{kHz}$. The same accounts for the $10 \mathrm{~Gb} / \mathrm{s} \mathrm{NRZ}$ signal, where the power penalty is also dominated by SBS although the degrading effects of SPM and SBS set in almost simultaneously. The results in Fig. 1 are supported by previous work, concluding that SBS can give rise to notable power penalties in $10 \mathrm{~Gb} / \mathrm{s}$ (NRZ) systems [5].

This common SBS/SPM dependency on launch power of both signal quality and subcarrier RF power is exploited for monitoring the excited non-linearities in the system. Fig. 2 illustrates the principle of operation: the subcarrier tone is inserted at the beginning of transmission and extracted down stream through a tap-off, or at the end of transmission. The detected tone RF power will then provide information about whether the signal has deteriorated due to excessive launch power. This information, which is otherwise unavailable, can provide operators with a valuable tool for both monitoring the signal quality and optimizing the launch power. Note that if the degradation from SBS is somehow eliminated (by e.g. phase dithering), the subcarrier power will still fade due to SPM (see Fig. 1). However, the fading will be less pronounced, which makes the scheme less attractive in this case.

\section{Field trial and results}

This method for monitoring excited non-linearities during transmission has been demonstrated in a lab environment and furthermore verified in a field-trial experiment over $500-\mathrm{km}$ of dark fiber in Denmark.

The single channel field trial experiment was conducted using a $5.1 \mathrm{GHz}$ tone with a modulation depth of $5 \%$ relatively to the $10 \mathrm{~Gb} / \mathrm{s}$ NRZ PRBS generated data. The trial was carried out in the part of the Danish Research Network depicted in the right part of Fig. 3. The three amplification sites, Lyngby, Næstved and Odense are marked out in the map with dots.
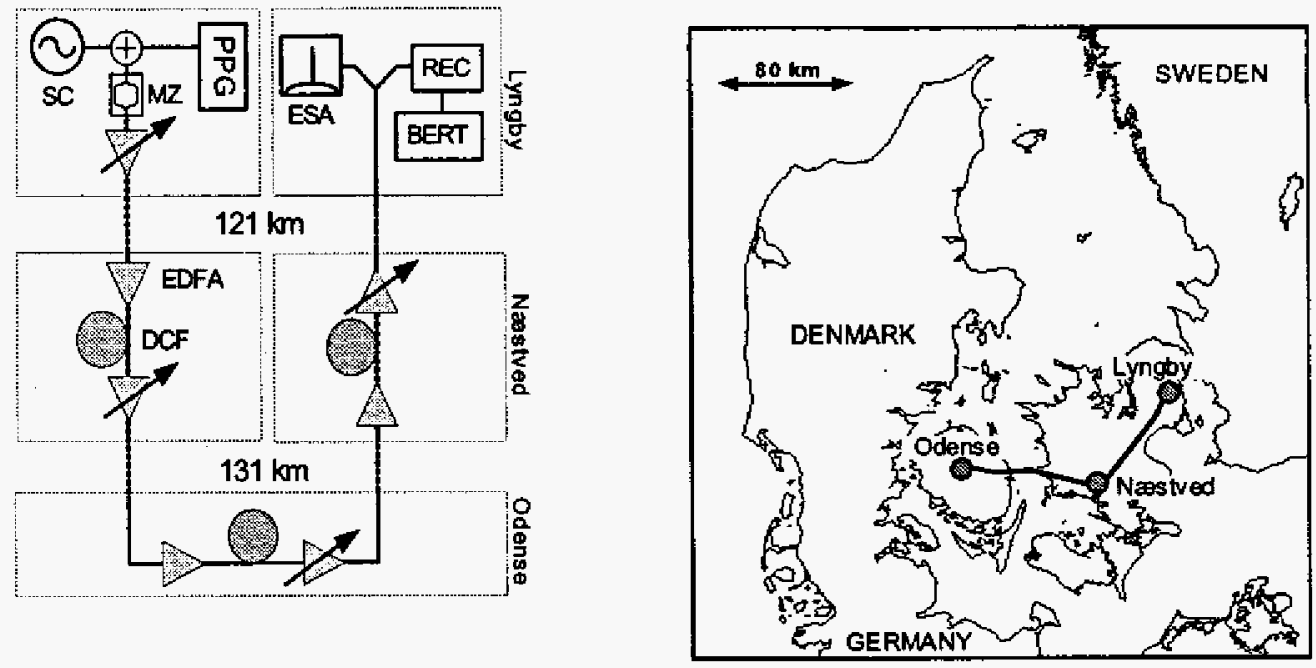

Fig. 3. Left: Figure illustrating the layout of the field-trial. The signal was sent from Lyngby via Nastved to Odense and looped back from Odense to Lyngby, again via Næstved. The total transmitted distance is $500-\mathrm{km}$ over four spans. Also depicted is the insertion and extraction of the subcarrier tone, which in turn is used for monitoring and optimization of the power. Right: A map that illustrates the transmission path in the dark fiber network. 


\section{OTuH2}

The fiber in the network complies with the G.652 ITU standard and has a dispersion parameter of $16-17 \mathrm{ps} / \mathrm{nm} / \mathrm{km}$. The total transmission distance of approximately $500-\mathrm{km}$ is broken up into four spans of $121-\mathrm{km}, 131-\mathrm{km}, 131-\mathrm{km}$ and $121-\mathrm{km}$, which is also evident from Fig. 3, where a simplified setup is shown in the left part of the figure.

In each re-amplification point, the signal was dispersion compensated as well as optically filtered. Each dispersion compensating fiber (DCF) section compensates close to $100 \%$ for the previous transmission span and, as illustrated in the left of Fig. 3, a double amplifier configuration was used. The first of the two amplifiers deliver a constant power of $0 \mathrm{dBm}$ to the DCF that follows. An attenuator follows the second amplifier in order to vary the launch power to the following transmission span.

Two scenarios were studied in the field-trial: a) Equal launch power to all four spans, varied from $+2 \mathrm{dBm}$ to +14 $\mathrm{dBm}$, and b) Launch power to the first span was varied within the same range and the rest remained unchanged. The reason for studying these two cases is that it might not be feasible or even possible to change the power to all spans in the transmission link. However, as will be evident from the results, this is the most desirable situation as a better signal quality can be achieved. In both the cases considered, the power to each span was changed and the variations of both the signal sensitivity and the subcarrier power were recorded.

Fig. 4 shows the results corresponding to scenario a), and it is clearly observed how the subcarrier power drops as the sensitivity is degraded. Analogously, Fig. 5 shows the results of scenario b), where the same tendency is seen although not as radical as in case a). Case a), where the input power is the same to all spans in the link, clearly exhibits a much more rapid change in both sensitivity and subcarrier power as the input power exceeds $\sim+11 \mathrm{dBm}$. This is intuitively expected as non-linearities will be excited throughout all the spans, whereas in case b) nonlinearities will primarily be excited in first span.

These results demonstrate the successful use of the non-linearity monitor in a practical and realistic scenario. With a miss-tuning of launch power of less than $2 \mathrm{~dB}$, the effect on the power penalty can be several $\mathrm{dB}$ 's. However, keeping track of the subcartier $R F$ power evolution this can be effectively avoided.

Several other span lengths and dispersion maps have been investigated in a lab environment, which display the same correlation between the signal quality and the subcarrier power.

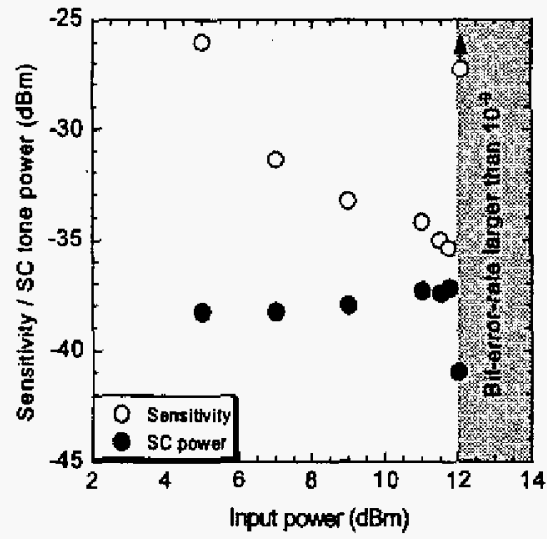

Fig. 4. Sensitivity versus subcarriet $R F$ power for case a) Equal launch power is launched to all four spans. Note the sharp drop in subcarrier power that is well synchronized to the sharp drop in signal quality.

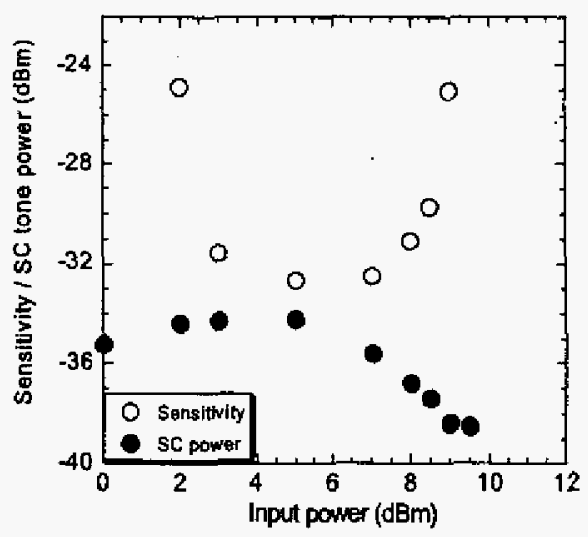

Fig, 5. Sensitivity versus subcarrier RF power for case b) Launch power to the first span was varied and the launch power to the remaining spans were fixed at $+7 \mathrm{dBm}$ into all spans.

\section{Summary}

This paper has presented a field-trial experiment documenting a new method of monitoring signal degrading nonlinearities in the fiber. By inserting a subcarrier into the baseband of the data signal before transmission, the level of detected RF power of the subcarrier will provide a measure for of the signal quality depending on launch power to the fiber. The field-trial results have showed the method to be simple and applicable for launch power optimization and non-linearity monitoring.

\section{References}

[1] T. Dimmick et al. "Optical Dispersion Monitoring Technique Using Double Sideband Subcarriers", PTL 14 (7), pp. 900-902, (2000).

[2] M.N. Petersen et al. "Dispersion monitoring and compensation using a single inband subcarrier tone" OFC 2001, Vol. 3, pp. WH4-1 (2001). [3] C.J. Youn "OSNR monitoritg technique based on orthogonal delayed-hemodyne method", PTL 14(10), pp. 1469-1471, (2002).

[4] M. Nezam et al. "First-Order PMD Monitoring for NRZ Data Using RF Clock Regenteration Techniques" ЛT, 2 (4), pp. 1086-1093, (2004).

[5] A.Sano et al."10 Gbit/s, $300 \mathrm{~km}$ repeaterless transmission with SBS suppression by the use of RZ.." Elec. Lett. 30(20), pp. 1694-95, (1994). 